\title{
1
}

\section{THE ALLERGIC ASPECTS OF URTICARIA AND ANGIONEUROTIC OEDEMA}

\author{
By GEORGE W. BRAY, M.B., M.R.C.P.
}

\author{
(Physician in Charge of Allergy Clinic and Children's Department, Prince of Wales' General \\ Hospital, Tottenham; Physician, Princess Elizabeth of York Hospital for Children, Shadwell)
}

- Urticaria and angioneurotic oedema are conditions in which whealing is the basic pathological condition, and, although all wheals are not necessarily allergic, the allergic mechanism is the most common one capable of producing whealing. The allergen affects the sensitive tissues in such a way that a diffusible histamine-like substance becomes available, producing local vascular or lymphatic changes, and thus eliciting the characteristic wheal.

\section{ALLERGY}

Symptoms are said to be of allergic origin when brought about by a patient showing an exaggerated susceptibility to some substance which is innocuous to ordinary normal individuals in the amounts commonly encountered. The agents provoking these reactions ("allergens") may be inhaled, ingested or injected; or may result from infection or contact. These agents may be living or inanimate, and repeated symptoms can only be brought about in each individual case by exposures to the same or to an immunologically related agent. The patients exhibiting these symptoms frequently belong to families where others suffer from asthma, hay fever, vasomoter rhinitis, migraine, eczema, urticaria and angioneurotic oedema; and the individual patient may suffer from several of these allergic symptoms. Pathologically they may all be explained by a smooth muscle spasm, a localised oedema, or a tissue infiltration with cells usually eosinophils. All respond temporarily to adequate doses of adrenaline injected.

\section{PATHOLOGY}

Cases of urticaria and angioneurotic oedema obey all these tenets, being explained by the localised oedematous type of allergic pathological reaction. In urticaria, the fluid accumulations occur solely in the skin and mucous membranes; in angioneurotic oedema or giant urticaria the subcutaneous tissues are also involved. In addition to the skin and subcutaneous tissues, visible swellings can be noted in the buccal, nasopharyngeal, laryngeal, bronchial, gastric, colonic and bladder mucosae, using appropriate instruments; and coincident with the skin and mucosal conditions symptoms indicating involvement of other organs may be experienced, as in the central nervous system causing migraine or fits, in the peripheral nervous system leading to paralysis, and even in the heart causing paroxysmal tachycardia. Presumably the cells in the walls of the blood-vessels and lymphatics are the shock tissues, the allergens being distributed after absorption through the respiratory and gastrointestinal tracts, and in some cases even by directly penetrating the skin's surface.

\section{CLINICAL APPEARANCES}

In the young, the urticarial type of rash is that usually experienced, rounded or irregular patches occurring which are firm and elevated, with whitish centres and erythematous borders, without definite arrangement and never symmetrical. The attacks may last a few hours to a few days, disappearing and reappearing with extreme rapidity, and usually accompanied by intense itching.

In the middle-aged, and more frequently in the female sex, the rash takes on the angioneurotic oedema type and swellings of such distensible tissues as the eyelids, lips, mouth, breasts, genitals and limbs may occur; variable in size, extent and shape. The lesions are usually raised, pale in colour, firm or elastic in consistency, and do not pit on pressure. They may be accompanied by such local sensations as pricking, burning, pain and itching, but more often merely by a feeling of tenseness. They often occur at night or on waking in the morning. 
Whealing may be produced by many different mechanisms either allergic or non-allergic:!

\section{Non-Allergic.}

Certain substances (Primarily Urticariogenic) may cause urticaria, in any individual, such as:-

A. Drugs.-Histamine and many alkaloids (morphine, codeine, atropine, pilocarpine, quinine) at injected sites.

B. Poisons. - The eating of certain decomposed foods containing poisonous ptomaines.

C. Animal.-The bites of insects, bugs; pediculi or leeches; the stings of bees, wasps, hornets, jellyfish or the Portuguese man-of-war; and the stinging hairs of woolly-bear caterpillars.

D. Vegetable.-The handling of nettles and hairy plants.

In these cases it is suggested that some intermediate diffusible substance is liberated or produced locally and acts directly on the blood vessels or lymphatics producing the wheal.

\section{Allergic}

Other substances may cause urticaria in sensitised individuals, and the sensitising substances (allergens) may be:-

A. Inhaled.-Commonly from feathers, down or horsehair in pillows, bolster, mattress and eiderdown; as a manifestation of pollen sensitivity during the hay fever season; in the presence of certain animals, dusts, smokes, powders or insecticides; and even in association with mould, fungi and yeast sensitivity.

B. Ingested.-(i) Foods. Commonly shellfish, fish, strawberries, cheese, nuts, eggs, wheat, milk, pork and chocolate. Alcohol is often incriminated, but only appears to act by aiding the absorption of some other food.

(ii) Drugs. Aspirin (salicylates), phenolphthalein, quinine, ipecacuhana, barbiturates, morphine, iodides, bromides, antipyrine, sulphanilamide.

C. Injected.-Following an overdose of allergen, serum injections or blood transfusion; resulting from the bites or stings of insects; or following therapy with drugs, vaccines, or such endocrine substances as insulin or even adrenaline.

D. Infectants.-Associated with septic infections (teeth, tonsils, nasal sinuses, intestine, gall-bladder, appendix, kidney and bladder); with intestinal parasites; or with mycotic infections of the skin, especially trichophytin.

E. Contactants.--In hypersensitive subjects the mere handling or lick of various animals, or even the use of their hair for bedding (horsehair), for furs (rabbit) or for clothing (wool, silk) causes whealing. Sometimes foods produce urticaria through external contact - as the barman whose hands swelled whenever he handled a lemon; the housewife whose hands and arms swelled when she handled flour; or the child whose lips swelled when touched with fish or egg-white, or even after being kissed by a mother who had eaten them recently. Reactions may also be caused by cosmetics (powders, nail varnish, lipstick, eye-black), dyes in clothing, various plants, occupational dusts, chemical solutions and even adhesive plaster.

F. Physical Agents.-(i) Light. Usually associated with the presence of photo-active substances in the blood;

(ii) Heat. Either applied to the skin or generated during work, exercise, excitement or febrile disease;

(iii) Cold. Either following its external application as in swimming, bathing or washing in cold water, or being out in a cold,wind or snow; or following its internal application, as after eating ices or drinking cold liquids;

(iv) Trauma. Where urticarial wheals appear at the site of rubbing, scratching, pressure or blows. 


\section{ASSOCIATED CONDITIONS}

The urticarial reactions often appear to be associated with endocrine disturbances or psychological states. Many women find their symptoms worse at the onset of the period, or at the time of the menopause. The conditions do not appear predominantly with any gross endoctine dysfunction, but are always more prevalent during lowered states of basal metabolism. Disturbances in the patient's psychological state may be the result of the skin trouble - the intense itching, the disturbance of sleep, the appalling disfigurement at times, and the apparent inefficiency of any immediate remedy; but it is more probable that under some psychological conditions the liberation of the histamine-like substance is facilitated. Urticarial outbursts frequently follow emotional storms, anger or fright, but they are usually in allergic persons, and the psychological upset utilises any available adrenaline, and renders the sufferer more susceptible to some offending allergen acting during this adrenaline exhaustion.

\section{DIAGNOSIS}

Though the common drugis causing whealing do not give positive skin tests, and persons exposed to certain occupational dusts may give positive skin tests without necessarily developing clinical symptoms on exposure, most cases of urticaria and angioneurotic oedema can be relieved by the taking of a careful history (having in mind the common groups of causes); the employment of skin tests; and the use of elimination measures in environment and diet.

It is among the ingested causes, the foods and drugs, that the lack of correlation between clinical symptoms and positive skin tests occurs, and it is possible that in its passage through the alimentary mucous membrane or wall, or at some point in the path from the mouth to the shock organ, the food or drug is split or altered by conjugation possibly with some protein substance; so the skin is sensitive only to the altered product as arriving at the shock tissues and not to the original substance ingested or to the substance used in the skin test.

\section{TREATMENT OF THE ACUTE ATTACK}

Inject subcutaneously five minims of adrenaline chloride solution $(1 / 1,000)$, and if the case is a severe one repeat the injection of a further three minims at $\frac{1}{4}, \frac{1}{2}, 1,2,4$ and 8 hours later as there will be a tendency for symptoms to reappear when the temporary effect of the adrenaline has worn off. The adrenaline action may also be sustained by injecting slow adrenaline (adrenaline in an oil, gelatin or glycerin base) or by inhaling the $1 / 100$ solution. If there is any oedema of the glottis larger and more frequent doses of adrenaline should be given and preparation made for intubation or tracheotomy to be performed.

Local irritation may be relieved by warm alkaline baths; the use of ordinary vinegar diluted with four parts of water; dusting with orris-root-free talcum powder containing $1 \%$ camphor and $\frac{1}{2} \%$ menthol; or employing a calamine lotion or cream containing $1 \%$ phenol and $1 \%$ menthol. The itching may also be lessened by giving ephedrine-half a grain four hourly, luminal, phenacetin or carbromal internally. Calcium by mouth or injection has no constant effect.

The gastro-intestinal tract should be cleared with an emetic if the case is seen early, and later saline aperients, castor oil or calomel will help. Food intake should be reduced and fluids forced. Alcohol should be forbidden as it hastens food absorption.

Factors increasing the blood supply to the skin, such as sudden violent exercise or overheating, should be avoided.

The administration of potassium chloride, histaminase (Torantil), or the giving of a high vitamin $C$ diet, have no constant beneficial effect, but the injection of ten units of insulin a day is sometimes helpful.

\section{TREATMENT TO PREVENT RECURRENCES}

Every effort should be made to determine the cause by a careful history, the use of skin tests, or the employment of eliminative measures in diet or environment.

In chronic cases the first thing to do is to stop all previous medication as drugs play such a large part in the causation of this condition, especially aspirin and phenolphthalein. Though 
chemical substances frequently cause clinical urticaria they hardly ever produce wheals on skin test.

Inhalant factors are the next commonest cause, especially in those cases appearing during the night or on waking in the morning due to a sensitivity to feathers, down or horsehair in the pillows, bolster, mattress or eiderdown. Other cases appear due to orris root or drugs in cosmetics, to occupational or house dusts, insecticides or animal hairs. These causes should be removed as far as possible, following skin tests, and desensitising measures are extremely beneficial

Any causative food should be avoided but in general foods are less common causes than the drug and inhalant groups. The treatment of flatulent indigestion or chronic gastritis with their concomitant hypochlorhydria is essential, and the administration of $20-30$ minims of dilute hydrochloric acid in a wineglassful of water immediately before each meal is indicated. The bowels should be kept open with saline aperients. Sometimes salol (10 grains thrice daily), ichthyol (5 minims in capsule thrice daily) or such adsorbants as charcoal or kaolin are beneficial.

Contactant substances may cause or irritate urticarial conditions, so the rôle of wool, silk, furs, dyed clothing, cosmetics, animals, adhesive plaster and occupational dusts needs consideration.

The physical causes form an important group. In light cases sunlight or ultra-violet light exposures are employed; in heat cases baths of gradually increasing temperature, exposures to diathermy or other electrical heat, or such pyrogenic therapy as B. Coli vaccine intravenously are sought: and in cold cases frequent cold baths, gradually decreasing the temperature whilst increasing the period of immersion, are utilised. Practically all cases due to physical agents may be benefited by a course of gradually increasing doses of histamine injected subcutaneously, beginning with $1 / 10 \mathrm{mg}$. and increasing daily by $1 / 10 \mathrm{mg}$. up to a dose of $1 \mathrm{mg}$.

In infective cases any septic foci should be eradicated as far as possible, and occasionally vaccines may prove of benefit.

In most cases there is an associated lowering of the basal metabolism and adequate doses of thyroid extract are necessary. In the presence of defective blood coagulability, calcium may help.

If the cause cannot be determined or removed, the withdrawal of $10 \mathrm{c} . \mathrm{cm}$. of blood from a vein at the elbow and its injection intramuscularly into the buttock each five days until six injections have been given is more uniformly successful than other non-specific methods such as injections of histaminase or peptone.

\section{SPECIAL NUMBERS OF THE JOURNAL}

Special numbers have been published from time to time, each dealing comprehensively with a particular branch of medicine or surgery. These special numbers are as follows, and copies, price 2s. each, post free, may be obtained from the Fellowship of Medicine, I Wimpole Street, London, W.I. A list of contents of any individual number will be sent on application.

\begin{tabular}{|c|c|c|}
\hline OBSTETRICS & (August, 1933) & Tropical Drseases \\
\hline OPHTHALMOLOGY & (August, 1934) & Cerebral Tumours \\
\hline NEUROLOGY & (April, 1935) & (February, 1940) \\
\hline UROLOGY & (November, I935) & *WaR Wounds OF the Chest \\
\hline CARDIOLOGY & (April, 1936) & *WaR Wounds of the ABDomen \\
\hline Proctology & (August, r936) & *WaR Wounds OF THE LIMBS (May, 1940) \\
\hline NEPHRITIS & (February, r937) & *War Wounds OF THE EYE AND ORBIT (June, 1940) \\
\hline RHEUMATISM & (September, 1937) & *WaR Wounds of the Head and Face (July, 1940) \\
\hline ORTHOPEDICS & (October, I937) & MEdicine IN THE U.S.S.R. (January, I942) \\
\hline
\end{tabular}

